\title{
Multi-channel Fourier Packet Transform of EEG: Optimal Representation and Time-Varying Coherence K. H. Ting ${ }^{1}$, M. Shen ${ }^{2}$, P. C. W. Fung ${ }^{1}$, F. H. Y. Chan ${ }^{1}$ \\ ${ }^{I}$ The University of Hong Kong, HONG KONG \\ ${ }^{2}$ Shantou University, CHINA
}

Abstract- Multi-channel recording of electroencephalogram (EEG) provides a measure of spatial-temporal pattern of cognitive processes. When oscillatory activities are going to be studied, the time-domain EEG signal can be analyzed via Fourier or wavelet transform. However the loss of temporal information after Fourier transform and the unavailability of phase information in wavelet transform limit their applicability in EEG analysis. In this paper, multi-channel Fourier packet transform is introduced. The algorithm resembles the wavelet packet transform by its binary tree search for an optimal selection of orthogonal basis, but extends the application to the multi-channel scenario. It aims to provide a sparse signal representation to localize features in the spatial-spectral-temporal domain. Since the decomposed atoms are spatially coherent components, analysis of time-varying synchrony across scalp locations is then possible.

Keywords - EEG, ERP, Fourier Packet, Coberence

\section{INTRODUCTION}

The traditional frequency-based analysis of EEG activities by partitioning frequency axis into delta, theta, alpha, beta and gamma bands has provided a useful tool for classification of mental states. For example, during eye-closed relaxation one exhibits prominent increase in alpha power. In early sleep stage, high amplitude delta wave will evolve. Apart from this, EEG coherence analysis, which is the measure of correlation between signals in the frequency domain, allows us to study how information flows through the brain. However transform of time-domain EEG data into frequency domain results in loss of temporal information. With the introduction of wavelet and time-frequency transforms, simultaneous analysis in time and frequency domain is then feasible.

By continuous wavelet transform (CWT) on individual EEG channel using complex kemels such as the Morlet wavelet, both the power and phase information in EEG can be extracted with desired temporal and spectral resolution. Combining the CWT of all the EEG channels will show how coherent the brain locations are and the phase relationship among them [1]. However it is computationally inefficient to evaluate CWT for highdensity EEG. Also it would be difficult to extract features from the transformed coefficients as they are highly redundant.

On the other hand, discrete wavelet transform (DWT) and wavelet packet transform (WP) allow fast decomposition of signal using orthogonal bases [2]. WP supports an optimal time-frequency tiling which is adaptive in nature. However both DWT and WP adopt real orthogonal bases and therefore no phase information can be obtained from the transformed coefficients.

In this paper, we introduce multi-channel decomposition using block Fourier basis. The objective of the decomposition is to seek for a sparse representation for the multi-channel signal such that spatially coherent spectral power is optimally localized in the time-frequency domain. Since Fourier basis is complex orthogonal basis, channel- to-channel phase difference can also be investigated from the transformed coefficients.

\section{METHODOLOGY}

A. Block Fourier Dictionary:

A block basis is constructed with Fourier basis of $\mathrm{L}^{2}[0,1]$ :

$$
\left\{e_{k}(t)=\exp (i 2 k \pi t)\right\}_{k \in Z}
$$

The time support of each block Fourier vector $g_{p, k}$ is $\left[a_{p}, a_{p+1}\right]$, of size $l_{p}$.

At each level of signal decomposition, the signal is partitioned in the time domain with equal interval by the dilated rectangular time window. Each partitioned signal can then be transformed by the orthogonal block Fourier basis. The rectangular window is dilated at each level to obtain different tiling in the time-frequency domain. At lower level, high spectral resolution but low temporal resolution will be obtained. At higher level, low spectral resolution but high temporal resolution will be obtained.

B. Best Orthogonal Basis

Combining the bases in all the level, the representation is over-complete. To look for the optimal basis, we would like to decompose the signal $x$ with orthogonal bases $\left\{e_{i}\right\}$,

$$
x=\sum_{i} \alpha_{i} e_{i}
$$

such that the entropy of decomposition coefficients is minimum

$$
\min \left\{I\left(\alpha_{i}\right)\right\}
$$

The entropy $I$ can be chosen as the $l^{l}$ norm of the decomposition coefficients,

$$
I\left(\alpha_{i}\right)=\sum_{i}\left|\alpha_{i}\right|
$$

The entropy minimization can be performed by an efficient binary tree search in a bottom-up manner [3]. The entropy at the nodes is compared across adjacent levels such that time partition will be merged if entropy at upper level is smaller than the lower level. At a result of the search process, an optimal tiling in the time-frequency domain can be found.

C. Extension to Multivariate Data

For multivariate data $\left\{x_{j}\right\}$, the decomposition for individual channel $j$ can be similarly expressed as

$$
x_{j}=\sum_{i} \alpha_{i, j} e_{i}
$$

with the global entropy measure being

$$
I\left(\alpha_{i}\right)=\sum_{i, j}\left|\alpha_{i, j}\right|
$$

Similar binary tree search can be performed resulting in a global optimal time-frequency tiling for all the channels.

\section{RESULTS AND DISCUSSION}

To demonstrate the proposed decomposition, we use ERP data captured by the 128-channel EEG system in an 
auditory oddball experiment. Subjects were presented with frequent (standard) tone at $1000 \mathrm{~Hz}$ and infrequent (target) tone at $1500 \mathrm{~Hz}$. Button press response was required for evaluation of correct stimulus classification. Fig.1 shows the averaged ERP for one of the subjects.
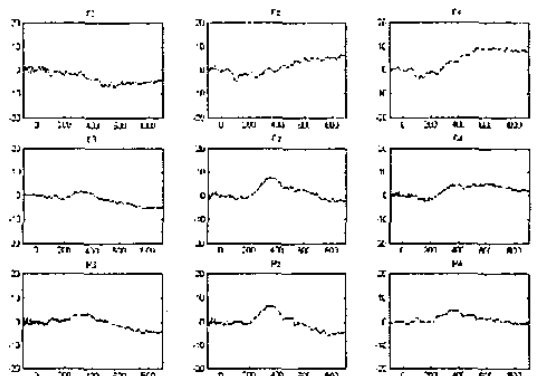

Fig. 1. Oddball ERP at electrodes F3, Fz, F4, C3, Cz, C4, $\mathrm{P} 3, \mathrm{Pz}$, and $\mathrm{P} 4$, average re-referenced.

The transform is performed on the averaged ERP, resulting in an optimal time-frequency tiling of the multichannel data as showed in Fig.2. The Heisenberg boxes in Fig. 2 represent not only the optimal time-frequency tiling for a single EEG channel, but for the whole 128 channels. Each box represents the exact coherent component among all the channels, since it is the same Fourier atom for all channels within a particular time interval and frequency. It is the transformed vector coefficient for each box that provides a measure of the synchronized power and phase distribution over the scalp.

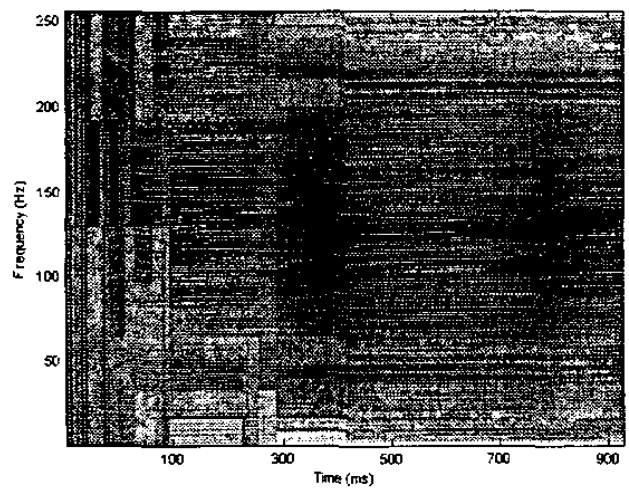

Fig.2. Heisenberg box showing the best time-frequency tiling for the multi-channel ERP epoch.

The vector coefficients for the boxes that exhibit significant power can be visualized as brain maps showing the distribution of coherent power and phase over the scalp. Fig. 3 and 4 show the maps for some DC and oscillatory components respectively. In Fig.3, the DC maps within two successive time intervals are shown. This is similar to traditional ERP topographic map which shows how activated brain regions propagate over time. Fig.4 renders the magnitude and phase distribution for a particular oscillatory component. Within the time interval, the magnitude map represents the exactly coherent power over the scalp at a particular frequency. How the coherent power flows across locations can be observed by the phase map.
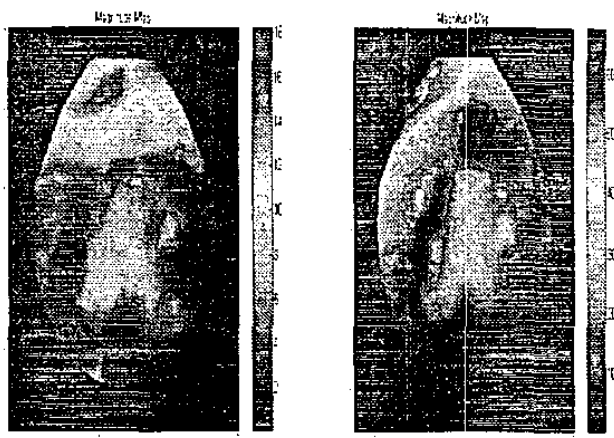

Fig.3. DC topographic map at (left) $256-284 \mathrm{~ms}$, (right) 284-412ms. (Eyes point upward.)
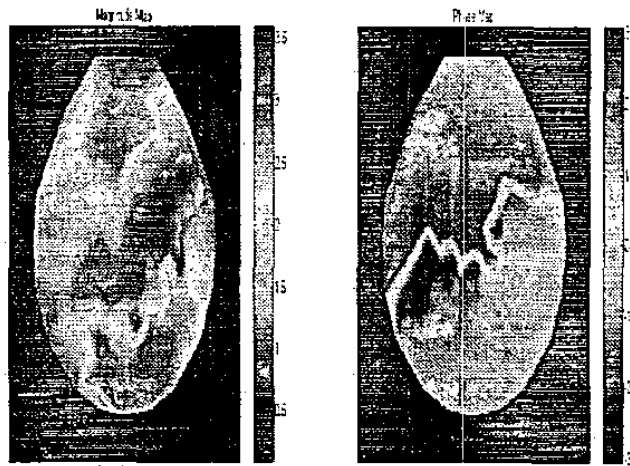

Fig.4. Topographic map for (left) magnitude and (right) phase distribution at $92-156 \mathrm{~ms}, 16 \mathrm{~Hz}$.

\section{CONCLUSION}

A multi-channel transform for EEG is constructed based on block Fourier basis and best orthogonal basis selection. Based on the proposed algorithm, an optimal timefrequency tiling for the multi-channel signal is possible. The aim is to seek for an optimal representation for the signal so that transformed coefficients are sparse in the spatial, spectral and temporal domain. Each vector of coefficients with respect to a particular atom in the dictionary then represents the coherent power across all the EEG electrode locations, and the phase relationship among them.

\section{REFERENCES}

[1] M. Shen, K. H. Ting, P. J. Beadle, F. H. Y. Chan, "Analysis of time-varying synchronization of EEG during sentences identification," Proc. $I^{\text {st }}$ Int. IEEE EMBS Conf. Neural Engineering, pp. 87-90, 2003.

[2] S. Mallat. A Wavelet Tour of Signal Processing. San Diego: Academic Press, 1999.

[3] R. R. Coifman and M. V. Wickerhauser, "Entropybased Algorithms for Best-Basis Selection", IEEE Trans. Information Theory, vol. 38, pp. 713-718, 1992. 